\title{
Group versus Individual Interpersonal Psychotherapy for Depressed Adolescents
}

\author{
Gabrielle O’Shea \\ University of Queensland, Brisbane, Australia \\ Susan H. Spence and Caroline L. Donovan \\ Griffith University, Brisbane, Australia
}

\begin{abstract}
Background: This study adds to the limited evidence concerning the benefits of Interpersonal Psychotherapy (IPT) with depressed adolescents. It evaluates the long-term effects of group versus individual delivery of this treatment approach. Aims: To conduct a smallscale examination of the long-term efficacy of group versus individual delivery of IPT for depressed adolescents. Method: Thirty-nine adolescents, aged 13-19 years, with a primary diagnosis of Major Depressive Disorder, were randomly assigned in blocks to either group or individual delivery of IPT. Standardized clinical interview and questionnaire assessments were conducted at pre- and posttreatment, and 12-month follow-up. Results: Intent-to-treat (ITT) analyses indicated significant improvements in depression, anxiety, youth-reported internalizing problems, and global functioning from pre- to posttreatment for those receiving IPT, with no significant differences in outcome between group and individual formats of delivery. Improvements were maintained at 12-month follow-up. Completer analyses also revealed significant and sustained improvements on these measures for those receiving IPT, with no differences in outcome between therapy formats for most measures. Individual IPT showed significantly greater improvements than group IPT in parent-reported internalizing problems for the completer but not the ITT analyses. Conclusions: Both individual and group formats of IPT offer promise in producing long-term benefits in the treatment of depression among adolescents.
\end{abstract}

Keywords: Interpersonal psychotherapy, adolescents, depression, group therapy.

\section{Background}

Research into the efficacy of interpersonal psychotherapy for depressed adolescents (IPT-A) is still in its infancy. The limited evidence to date suggests that IPT-A, delivered on an individual basis, significantly reduces depressive symptoms compared to a wait-list (Rosselló and Bernal, 1999), a clinical monitoring condition (Mufson, Weissman, Moreau and Garfinkel,

Reprint requests to Susan H. Spence, Australian Institute of Suicide Research and Prevention, Griffith University, Brisbane, Australia 4122. E-mail: s.spence@ griffith.edu.au 
1999), and treatment-as-usual (Mufson, Dorta et al., 2004), with effects equivalent to cognitive behaviour therapy (Rosselló and Bernal, 1999).

Mufson, Gallagher, Dorta and Young (2004) and Rosselló, Bernal and Rivera-Medina (2008) subsequently adapted IPT-A for use in a group format. Group versus individual therapy approaches have their relative advantages and disadvantages. Individual IPT has the advantage of enabling the therapist to give greater attention to individual issues, to monitor client progress more stringently, and to adapt the intervention to particular issues relevant to the client. In contrast, a group format provides an opportunity for young people to realize that they are not alone in experiencing depression, to learn from the experience of others, to benefit from mutual support, and to observe and practise interpersonal and problem solving skills in the session (Mufson, Gallagher et al., 2004). If effective, the group format has the obvious advantage of being cheaper to deliver in terms of therapist time per client.

We could identify only one published randomized controlled trial comparing the efficacy of group versus individual IPT-A and this reported no significant differences between formats (Rosselló et al., 2008). This is an important finding given the need to develop cost-efficient methods of mental health service delivery, particularly for adolescents who are typically an under-serviced population (Kieling et al., 2011). It should be noted that the program developed by Rosselló and colleagues differs from the original IPT-A intervention developed by Mufson and her team (Mufson, Moreau, Weissman and Klerman, 1993) in that it was adapted to take into account the Puerto Rican cultural context.

\section{Aims and hypotheses}

The aim of the present study was to add to the limited evidence to date by conducting a small-scale evaluation of the relative efficacy of group versus individually administered IPTA. It provides an advance to knowledge by including a 12-month follow-up, as no long-term follow-up was conducted by Rosselló et al. (2008). It also included a clinical standardized interview to provide diagnostic data, rather than relying on self-report questionnaire data as was the case in the Rosselló et al. (2008) study.

The first hypothesis was that adolescents receiving both group IPT (IPT-A-GRP) and individual IPT (IPT-A-IND) would improve significantly from pre- to posttreatment on measures of diagnostic status for major depressive disorder, depressive symptoms, anxiety symptoms, internalizing problems more broadly, number of comorbid diagnoses, and global functioning. No significant differences in outcome between group and individual formats were predicted at posttreatment.

The second hypothesis predicted that, over the 12-month follow-up period, adolescents in the individual treatment condition would be more likely to retain therapeutic improvements than those in the group delivery approach. We proposed that individual therapy, with the capacity for more individual attention from the therapist and greater capacity to tailor the intervention to the needs of the client, would result in more sustained treatment outcomes over the 12-month follow-up period.

Ideally, a study of this type would include a waitlist control condition to ensure that both treatment approaches were superior to no-intervention. However, a waitlist control condition with a vulnerable adolescent population creates significant ethical issues. Initial pilot work involving a waitlist control condition indicated that parents, General Practitioners and school staff were highly reluctant to permit young people in their care to be allocated to a waitlist 
condition. As such, the present study was limited to a randomized controlled trial involving two clinical treatment conditions, namely group versus individual IPT-A.

\section{Method}

\section{Participants}

The sample included 39 adolescents aged 13 to 19 years $(M=15.33, S D=1.37)$ with 6 males and 33 females attending one of 19 schools in the Brisbane metropolitan area, Australia. Referrals came from school guidance officers, other mental health professionals, teachers and parents in response to information booklets about the treatment program and the nature of depression in young people. All had a primary diagnosis of Major Depressive Disorder (MDD) as determined by the Schedule for Affective Disorders and Schizophrenia for SchoolAge Children - Epidemiological version, $5^{\text {th }}$ edition (K-SADS-E; Puig-Antich and Chambers, 1978). Exclusion criteria, identified from the screening interview or pretreatment assessment, were: undergoing psychological or pharmacological treatment for depression currently or in the past month; currently reporting suicidal intentions or severe ideation; a Bipolar I or II diagnosis; a history of chronic medical illness or psychosis; or significant developmental delay.

Following a parent-completed telephone screening interview, adolescents and their parents were invited to participate in the program, to provide written informed consent from both a parent and young person, and to complete the K-SADS-E interview (youth only). Adolescents who did not meet criteria were excluded from the study and, where appropriate, were provided with other referral options. In addition to a primary diagnosis of MDD, 23 of 39 (58.9\%) participants were diagnosed with at least one comorbid diagnosis, and 10 of 39 (25.6\%) with two or more comorbid diagnoses. Specifically, $16(41 \%)$ presented with one or more anxiety disorder(s), 9 (23\%) with alcohol or substance abuse, $2(5 \%)$ with oppositional defiant or conduct disorder, $3(8 \%)$ with attention deficit hyperactivity disorder, and $2(5 \%)$ with bulimia nervosa.

Seventy-six percent of the sample were born in Australia, $51.3 \%$ were living in families with both biological parents, $23.1 \%$ in single-parent families, $20.5 \%$ with a parent and stepparent, and $5.1 \%$ were living out of home. One adolescent identified as Aboriginal, with the remainder being of Caucasian ethnic origin. In terms of annual family income, $26.1 \%$ reported earnings of less than AUD \$40,000; $41 \%$ between AUD \$40,000 and AUD \$69,000; and $25.6 \%$ more than AUD $\$ 70,000$ per annum. These data suggest that, on average, the adolescents came from low to middle-income Australian families.

Figure 1 presents a flow diagram of participant progression through the different phases of the study. Sixty-three adolescents were recruited to the program. Of those meeting screening criteria, $17(27 \%)$ were excluded for a range of reasons including failure to complete the assessments $(n=2)$, no further interest in participating prior to interviews $(n=4)$, nonclinical levels of depression $(n=7)$, presence of a developmental disorder $(n=1)$, presence of a primary behavioural disorder $(n=1)$, and recently commenced anti-depressant medication $(n=2)$. A further 7 families declined to participate despite meeting eligibility criteria, but before being allocated to their randomized condition. Refusal was usually because of the time commitment required. 


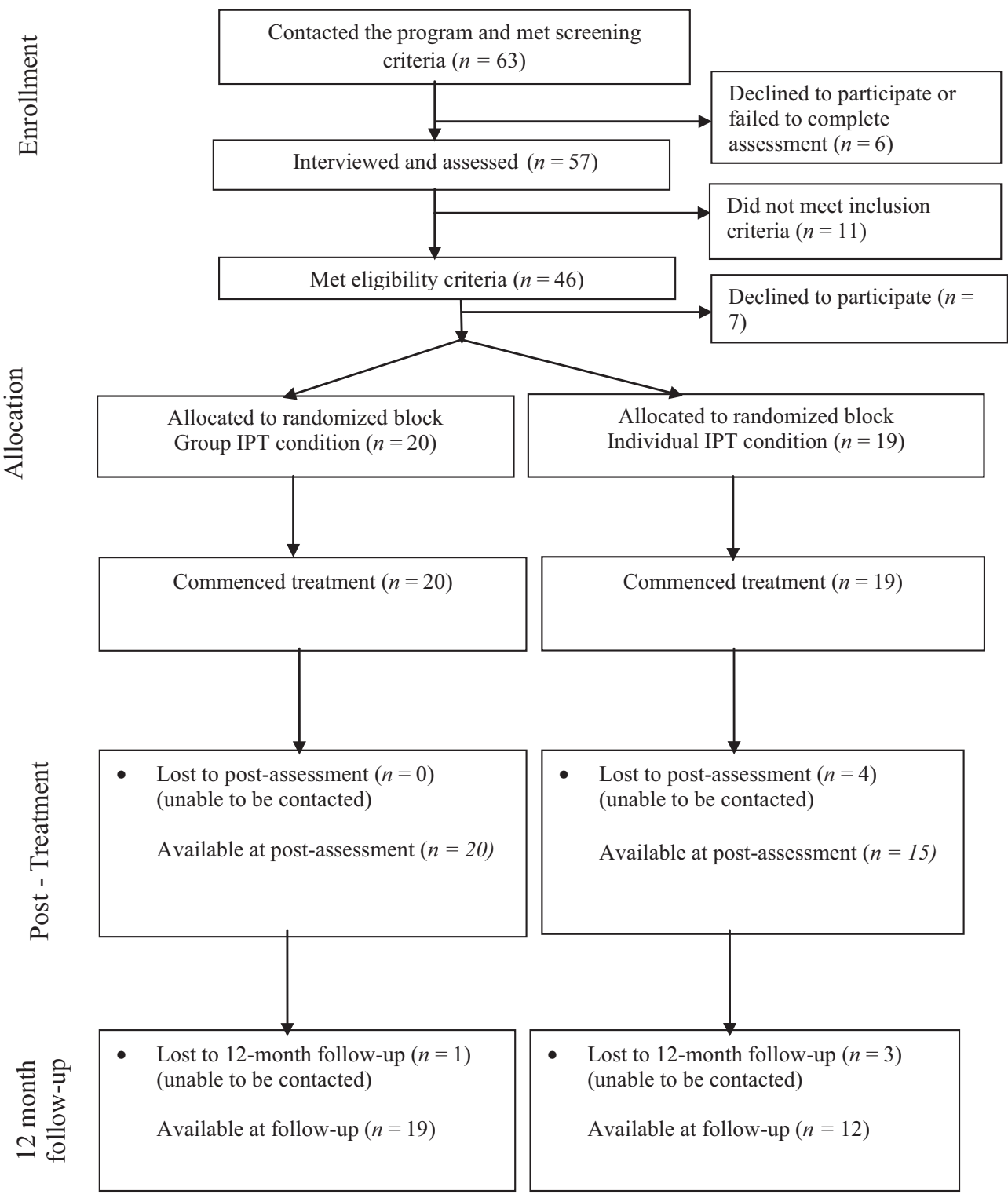

Figure 1. Flow diagram of participants' progress through the study

After determining eligibility and reconfirming willingness to participate, 39 participants were allocated to a randomized block, with 20 being allocated to group IPT (IPT-A-GRP) and 19 to individual IPT (IPT-A-IND). Block randomization, in blocks of 6 to 8 participants, was used in order to ensure sufficient numbers to start the group therapy condition as quickly as possible after assessment. Recruitment efforts were phased to ensure sufficient numbers to fill 
the groups at specific time points without long wait periods. On average, participants in both conditions waited for an average of around 2 weeks to commence treatment after completion of assessments. All participants attended more than $75 \%$ of therapy sessions. Four IPT-AIND participants failed to provide posttreatment and 12-month follow-up data. A further three from IPT-A-IND and one from IPT-A-GRP did not provide 12-month follow-up data. Reasons for missing data related to families having moved house or failure to respond to attempts at contact.

\section{Measures}

Diagnostic interviews (youth) and questionnaires (parent and youth) were completed at pretreatment, immediately following treatment (12-weeks post-baseline), and 12-months posttreatment.

The Schedule for Affective Disorders and Schizophrenia in School-Age Children Epidemiological Version $-5^{\text {th }}$ Revision (K-SADS-E; Puig-Antich and Chambers, 1978) was used to determine diagnostic status. The K-SADS-E is a structured interview assessing psychological disorders in children and adolescents aged 6 to 18 years according to the Diagnostic and Statistical Manual of Mental Disorders-IV (DSM-IV; American Psychiatric Association, 1994). It has strong psychometric properties, with an inter-rater agreement of .73 and a test-retest reliability of .90 (Kaufman, Birmaher, Brent and Rao, 1997; Klein, Dougherty and Olino, 2005). Interviewers were blind to the experimental condition of the participants and completed training by the researchers until they reached a satisfactory level of competency, as judged by the first or second author, before completing assessments for the study. Training included familiarization with the instrument, observation of videotaped demonstrations, and supervised practice with observation and feedback of role-played and/or actual cases. Interviewers were psychology Honours graduates, all but one of who were completing, or had completed, Masters or Doctoral degrees in clinical psychology. It was beyond the resources of the current study to record the interviews in order to conduct independent assessment of inter-assessor agreement. However, interviewers participated in regular supervision of interviews throughout the study to increase the reliability of diagnoses.

The Beck Depression Inventory - II (BDI-II; Beck, Steer and Brown, 1996) assessed youth self-report of depressive symptoms. Total scores range from 0 to 63 , with higher scores indicating higher levels of depressive symptoms. The BDI-II has been identified as a sensitive instrument for detecting depressive symptoms in adolescents (Atlas and DiScipio, 1992), with high internal consistency (Ambrosini, Metz, Bianchi and Rabinovich, 1991; Barrera and Garrison-Jones, 1988; Kumar, Steer, Teitelman and Villacis, 2002; Strober, Green and Carlson, 1981), and good convergent validity (Barrera and Garrison-Jones, 1988).

The Children's Global Assessment Scale (CGAS: Shaffer et al., 1983) was completed by the independent interviewers, based on the K-SADS-E clinical interview, to provide an assessment of general functioning. CGAS values range from 1 (the greatest level of functional impairment) to 100 (the highest level of functioning), with scores between 81-100 indicating a normal level of functioning, scores between 61-80 indicating slight disability, scores between 41-60 indicating moderate disability, and scores between 1-40 indicating serious disability (Shaffer et al., 1983). The CGAS has high reliability between raters and across time (Shaffer et al., 1983). 
The Youth Self-Report-Revised (YSR; Achenbach, 1991) and parent-report on the Child Behaviour Check List (CBCL; Achenbach, 1991) assessed internalizing problems more broadly. These widely used measures of behavior problems have well-established psychometric properties (Achenbach, 1991; Achenbach, Dumenci, and Rescorla, 2002) and yield a total behaviour problem score and internalizing and externalizing subscales. The internalizing subscales (YSR-Int and CBCL-Int) were used in the present study, with scores ranging from $0-62$ and $0-56$ respectively.

The Spence Children's Anxiety Scale (SCAS-C; Spence, 1998) was included to measure anxiety symptoms given the high co-morbidity between anxiety and depression. The SCAS$\mathrm{C}$ assesses adolescents' perceptions of the frequency with which they experience anxiety symptoms. Scores range from 0 to 114 , with higher scores reflecting higher anxiety. The SCAS-C has high internal consistency and good convergent validity with other measures of anxiety (Muris, Schmidt and Merckelbach, 2000; Spence, 1998; Spence, Barrett and Turner, 2003).

The number of comorbid diagnoses was assessed from the K-SADS-E to provide an indication of the impact of intervention upon other aspects of mental health. The K-SADS$\mathrm{E}$ assesses a broad range of mental health problems including anxiety disorders, eating disorders, substance abuse, attention deficit hyperactivity disorder and conduct disorders, in addition to depressive disorders.

\section{Content of the intervention}

Treatment took place at either the School of Psychology Clinic, University of Queensland or in the counseling services facilities of a State High School, with both individual and group conditions being conducted at each setting. The delay, on average, between pretreatment assessment/diagnostic interview and starting treatment was around 2 weeks for both individual and group treatments. The study was conducted in compliance with the University of Queensland Human Ethics Committee.

Individual treatment condition. Interpersonal psychotherapy (IPT) was developed in the 1970s by Klerman and colleagues (Klerman, Dimascio, Weissman, Prusoff and Paykel, 1974) as an intervention to treat depression in adults. It aimed to reduce depression by building social support and enhancing the quality of interpersonal relationships, and assisting individuals to manage interpersonal conflicts, transitions and losses in an effective way. Subsequently, the approach was adapted by Mufson et al. (1993) for use with depressed adolescents. The IPT-A-IND sessions followed a detailed protocol adapted from Mufson et al. (1993) and comprised 12 sessions, conducted once per week over 12 weeks, with sessions lasting 50-60 minutes, with one therapist to each client. Four maintenance sessions were provided during the 12-month follow-up period.

Treatment included three main phases. The first phase consisted of four sessions, the first two of which aimed to identify and clarify the adolescent's interpersonal difficulties in one or more principal problem areas (interpersonal disputes, interpersonal sensitivities, interpersonal role transitions, grief and single parent family situations) using the Interpersonal Inventory with the adolescent to detail features of his or her interpersonal environment and any events associated with the onset of depression. The therapist explained the theoretical rationale for IPT-A, outlined the role of the adolescent in the therapy process, and together the teenager and 
therapist set a treatment contract and clarified therapy goals. Sessions 3 and 4 continued the first phase and focused on identifying links between specific interpersonal situations and low mood and depression, clarifying the principal problem area(s), identifying the communication patterns of those involved, and beginning to discuss alternative ways of responding.

The middle phase included sessions 5 to 9 , which focused on the particular interpersonal problems identified by participants, exploring the adolescent's perceptions and expectations relating to those situations, and assisting the young person to develop strategies and skills for more effective management of interpersonal problem situations. Therapy techniques in this phase included development of problem solving and interpersonal skills through discussion of the situation and alternative responses, analysis of communication patterns, rehearsal of skills in sessions, practice at implementing a proposed solution in interpersonal situations, and monitoring of, and providing feedback regarding, attempted solutions. Sessions 10 to 12 were focused on the termination phase, including anticipating future problems, putting in place contingency plans for future treatment, and encouraging the young person to feel a sense of mastery over the targeted problems, in addition to consolidation of skills for managing interpersonal issues.

While the session protocol drew strongly from that described by Mufson et al. (1993), adjustments were made from the original protocol so that individual and group delivery formats could be closely aligned and so that issues outlined by Mufson, Gallagher et al. (2004) for conducting IPT in a group format could be taken into account. In the present study, the diagnostic interview was completed prior to commencement of treatment rather than being embedded in the first four sessions. The middle phase was extended to include sessions 5 to 9 rather than sessions 5 to 8 , with the termination phase taking place in sessions 10 to 12 rather than 9 to 12 .

To reduce the risk of relapse, maintenance sessions were conducted on an individual basis at 1, 3, 6, and 12-months after completion of treatment. Maintenance sessions focused on checking progress, identifying current problems, and reviewing and rehearsing the strategies and skills for managing interpersonal issues learned during the treatment sessions.

Group therapy condition. The content of the IPT-A-GRP sessions closely mirrored the IPT-A-IND sessions but was adapted for group delivery taking into account the recommendations of Mufson, Gallagher et al. (2004) and Wilfley and colleagues (Wilfley, MacKenzie, Welch, Ayres and Weissman, 2000). It included the same order of session content, the same topics, and the same number of sessions over the same treatment period, including the maintenance sessions. However, group sessions lasted approximately 90 minutes to accommodate group discussion of individual group member issues. Each IPT-A-GRP session was conducted with groups of 6-8 adolescents. The first two sessions of IPT-AGRP were conducted on an individual basis as described by Mufson, Gallagher et al. (2004), in the same manner as IPT-A-IND, as this first phase is not conducive to group delivery. These sessions also prepared the adolescent for group participation. Sessions 3 and 4 in IPTA-GRP were conducted in a group format. These sessions continued the initial treatment phase as outlined for IPT-A-IND but also included content relating to establishing the group, encouraging group members to get to know each other, setting rules about group interaction and confidentiality, sharing of experiences and feelings, and providing positive feedback to each other. The remaining group sessions mirrored the IPT-A-IND content, with sessions 59 reflecting the middle phase of treatment outlined above, and sessions 10 to 12 being the 
termination phase. Maintenance sessions were also conducted at 1, 3, 6, and 12-months after the end of treatment, with content mirroring that of the individual approach, but delivered in a group format.

\section{Therapists and training}

Treatment was conducted by five psychologists who were trained in IPT methods using the training approach outlined by Rounsaville and colleagues (Rounsaville, Chevron, Weissman, Prussof and Frank, 1986). Attempts were made to counterbalance therapists across conditions but this was only partially completed. Therapists were assigned such that the first author conducted two of the three groups and around 50\% of individual cases, a second therapist conducted the third group and a small number of individual cases, and the other three therapists completed the remaining 6 individual cases between them. Training involved thorough familiarization with the IPT-A manual (Mufson et al. (1993), seminar format discussion of the manual and videotaped examples, and supervised case-work with four cases. The lead therapist had attended a number of intensive IPT training sessions conducted by a senior IPT expert. Ongoing supervision of casework through review of and feedback from weekly videotapes, was provided for each therapist by a senior, experienced IPT practitioner who was independent of the study and research group. This process provided feedback to each therapist regarding their performance against a modified version of the Interpersonal Therapy Scale from the Collaborative Study Psychotherapy Rating Scale (Hill, O'Grady and Elkin, 1992) to enhance treatment fidelity. Refresher training was conducted every 6 months.

\section{Results}

\section{Pretreatment comparisons}

There were no significant pretreatment differences between the two conditions in terms of adolescents' gender, ethnic background, home environment, age, parents' age, marital status, or parental occupation. There was however, a significant difference between the conditions for family income, which was lower in the IPT-A-GRP (10\% with family income over AUD $\$ 70,000)$ condition than the IPT-A-IND condition $(42.1 \%$ with family income over AUD $\$ 70,000), F(1,39)=4.9, p=.03, \eta^{2}=.12$. Although there were more males in IPT-A-GRP (5 of $20,25 \%)$ than IPT-A-IND ( 1 of $19,5.3 \%$ ) this difference was not statistically significant, $\chi^{2}(1, N=39)=2.92, p=.18$.

With regard to diagnostic variables, no significant pretreatment differences across conditions were found for the BDI-II, CGAS, SCAS-C, CBCL-Int, YSR-Int, or number of co-morbid diagnoses (See Table 1). In the IPT-A-GRP condition, 12 of 20 (60\%) met criteria for a pretreatment comorbid diagnosis, with 7 (35\%) being diagnosed with one or more anxiety disorder(s), 1 (5\%) with bulimia nervosa, 6 (30\%) with alcohol or substance abuse, 2 $(10 \%)$ with attention deficit hyperactivity disorder, and $2(10 \%)$ with conduct disorder. In the IPT-A-IND condition, 11 of $19(58 \%)$ met criteria for a comorbid diagnosis, with $9(47 \%)$ being diagnosed with one or more anxiety disorder(s), 1 (5\%) with bulimia nervosa, 3 $(16 \%)$ with alcohol or substance abuse, and $1(5 \%)$ with attention deficit hyperactivity disorder. 
Table 1. Values for diagnostic status and outcome variables for the ITT sample across time and condition

\begin{tabular}{|c|c|c|c|c|c|c|c|}
\hline \multirow{2}{*}{$\begin{array}{l}\text { Treatment condition } \\
\text { Depression measures }\end{array}$} & \multirow[b]{2}{*}{ Time } & \multicolumn{3}{|c|}{ IPT-A-GRP } & \multicolumn{3}{|c|}{ IPT-A-IND } \\
\hline & & $n$ & $N$ & $\%$ & $n$ & $N$ & $\%$ \\
\hline \multirow[t]{4}{*}{ Free of MDD diagnosis } & Pre & 20 & 0 & 0 & 19 & 0 & 0 \\
\hline & Post & 20 & 18 & 90.0 & 19 & 14 & 73.7 \\
\hline & 12-mth follow-up & 20 & 16 & 80.0 & 19 & 14 & 73.7 \\
\hline & & $n$ & $M$ & $S D$ & $n$ & $M$ & $S D$ \\
\hline \multirow[t]{3}{*}{ BDI-II } & Pre & 20 & 27.51 & 9.20 & 19 & 24.33 & 10.22 \\
\hline & Post & 20 & 17.57 & 13.79 & 19 & 14.00 & 12.45 \\
\hline & 12-mth follow-up & 20 & 13.14 & 13.77 & 19 & 13.37 & 11.01 \\
\hline \multirow[t]{3}{*}{ CGAS } & Pre & 20 & 51.90 & 10.95 & 19 & 48.47 & 11.96 \\
\hline & Post & 20 & 66.95 & 13.47 & 19 & 70.47 & 19.25 \\
\hline & 12-mth follow-up & 20 & 72.00 & 19.49 & 19 & 66.32 & 18.83 \\
\hline \multirow[t]{3}{*}{ CBCL-Int } & Pre & 20 & 18.80 & 8.17 & 19 & 18.74 & 8.81 \\
\hline & Post & 20 & 18.14 & 7.85 & 19 & 13.79 & 8.02 \\
\hline & 12-mth follow-up & 20 & 16.83 & 9.18 & 19 & 10.65 & 7.60 \\
\hline \multirow[t]{3}{*}{ YSR-Int } & Pre & 20 & 27.10 & 11.01 & 19 & 25.74 & 10.35 \\
\hline & Post & 20 & 20.85 & 11.27 & 19 & 17.32 & 12.58 \\
\hline & 12-mth follow-up & 20 & 18.55 & 13.87 & 19 & 18.45 & 11.51 \\
\hline \multirow[t]{3}{*}{ SCAS } & Pre & 20 & 41.36 & 13.74 & 19 & 47.65 & 13.87 \\
\hline & Post & 20 & 35.61 & 14.34 & 19 & 37.70 & 13.93 \\
\hline & 12-mth follow-up & 20 & 34.98 & 19.89 & 19 & 36.84 & 10.62 \\
\hline \multirow[t]{3}{*}{ Comorbid diagnoses } & Pre & 20 & 1.05 & 1.15 & 19 & 0.84 & 0.92 \\
\hline & Post & 20 & 0.50 & 0.95 & 19 & 0.47 & 0.77 \\
\hline & 12-mth follow-up & 20 & 0.45 & 1.05 & 19 & 0.42 & 0.77 \\
\hline
\end{tabular}

Notes:BDI-II = Beck Depression Inventory - II; CBCL-Int = Child Behaviour Checklist -Internalizing subscale; YSR-Int = Youth Self Report - Internalizing subscale; SCAS = Spence Children's Anxiety Scale; CGAS = Children's Global Assessment of Functioning; IPT-A-GRP = Group delivery of IPT for adolescents; IPT-A-IND = Individual delivery of IPT for adolescents

\section{Analyses}

Treatment effects were analysed for the intent-to-treat (ITT) sample, for each continuous variable separately, using repeated measures ANOVAs first from pre- to posttreatment and then from posttreatment to 12-month follow-up. This process was then repeated for the completer sample. The pre- to posttreatment analyses for the completer sample included all participants providing data on both of these occasions. Similarly, the posttreatment to 12-month follow-up analyses were restricted to those providing data at both of these time points. Given the significant pretreatment difference in family income, the data were also analysed using repeated measures ANCOVAs with family income entered as a covariate. Given that these results paralleled the ANOVA results, only the ANOVA results are presented below. Analyses also showed that there were no significant differences for any of the outcome measures across sites for either ITT or completer samples. Where appropriate, an indication of effect size is presented using partial eta-squared $\left(\eta^{2}\right)$, the proportion of the total variance 
that is attributed to an effect, with values ranging from 0 to 1 . Chi-square analyses were used for the categorical diagnostic variables.

\section{Intent-to-treat sample}

For the intent-to-treat (ITT) analyses $(N=39)$, missing data were replaced using the last observation carried forward (LOCF) method.

Pre- to posttreatment. As shown in Table 1, 18 of 20 (90.0\%) in the IPT-A-GRP condition and 14 of $19(73.7 \%)$ in the IPT-A-IND condition no longer met criteria for MDD at posttreatment, with no significant difference between conditions, $\chi^{2}(1, N=39)=1.76, p=$ .23. Univariate analyses revealed a significant time effect from pre- to posttreatment for the BDI-II, $F(1,37)=24.14, p<.001, \eta^{2}=.395$, CGAS, $F(1,37)=65.59, p<.001, \eta^{2}=.639$, YSR-Int, $F(1,37)=24.40, p<.001, \eta^{2}=.397$, CBCL-Int, $(1,37)=6.85, p=.013, \eta^{2}=$ .156 , SCAS-C, $F(1,37)=16.64, p<.001, \eta^{2}=.310$, and number of comorbid diagnoses, $F(1,37)=79.90, p<.001, \eta^{2}=.68$, with no significant condition by time or condition effects. Although Table 1 suggests that reductions on the CBCL-Int were greater for the IPTA-IND condition than the IPT-A-GRP condition, the time by condition effect just failed to reach statistical significance, $F(1,37)=6.84, p=.053, \eta^{2}=.10$. Figures $2 \mathrm{a}$ and $2 \mathrm{~b}$ show the mean change over time for the BDI-II and CGAS respectively for the ITT sample.

Posttreatment to 12-month follow-up. At 12-month follow-up, 16 of $20(80.0 \%)$ in the IPT-A-GRP condition and 14 of $19(73.7 \%)$ in the IPT-A-IND condition no longer held a MDD diagnosis, again with no significant difference between conditions, $\chi^{2}(1, N=39)=$ $0.22, p=.72$. From posttreatment to 12 -month follow-up there were no significant time, condition, or condition by time effects effect for the BDI-II, CGAS, YSR-Int, SCAS-C, or number of comorbid diagnoses (see Table 1 and Figures $2 \mathrm{a}$ and $2 \mathrm{~b}$ ). Where improvements had been made from pre- to posttreatment, they were maintained at 12-month follow-up. For the CBCL-Int there were no significant condition or condition by time effects, but there was a significant effect for time, $F(1,37)=4.45, p=.042, \eta^{2}=.107$, with participants in general tending to show improvements on this measure during the follow-up period.

Taken together, the ITT results suggest that adolescents who received IPT demonstrated significant reductions in depression, youth reported internalizing problems and anxiety, and increases in global functioning from pre- to posttreatment, with no significant differences between group and individual formats. Improvements from pre- to posttreatment tended to be maintained at 12-month follow-up, with no significant differences in maintenance effects between individual and group treatment conditions.

\section{Completer analysis}

ITT analyses are inherently conservative as the estimates of missing data are based on the assumption that individuals who are unavailable for reassessment have remained unchanged over time. In contrast, completer analyses included participants who not only commenced their allocated condition but who also participated in treatment and completed the relevant posttreatment or follow-up assessment of outcome. In the present study, all participants had completed at least $75 \%$ of sessions. Results are shown in Table 2. 
(a)

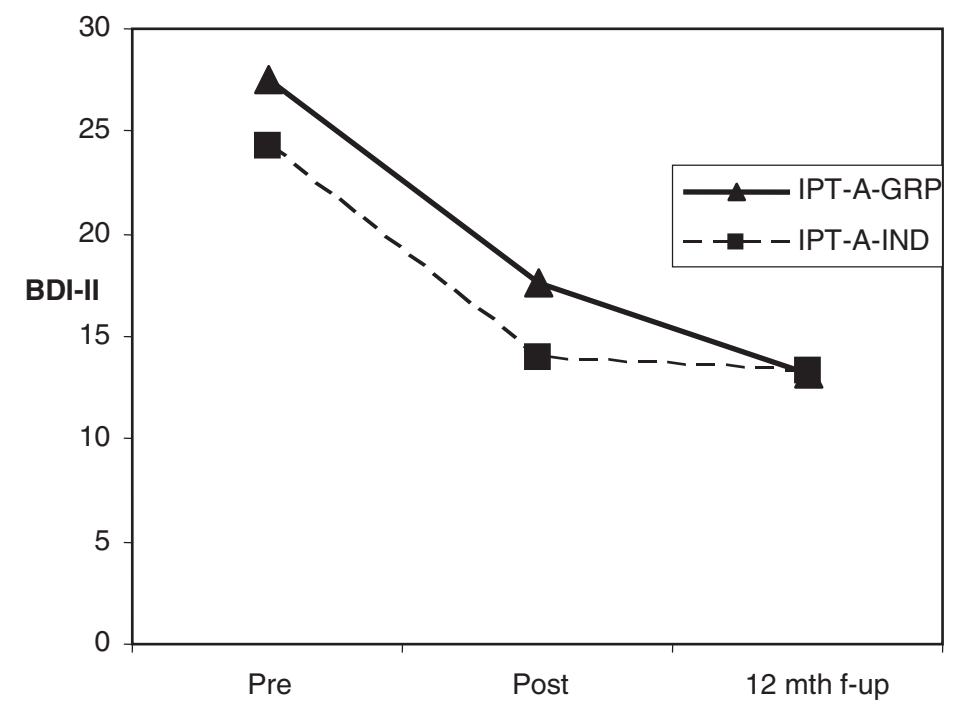

(b)

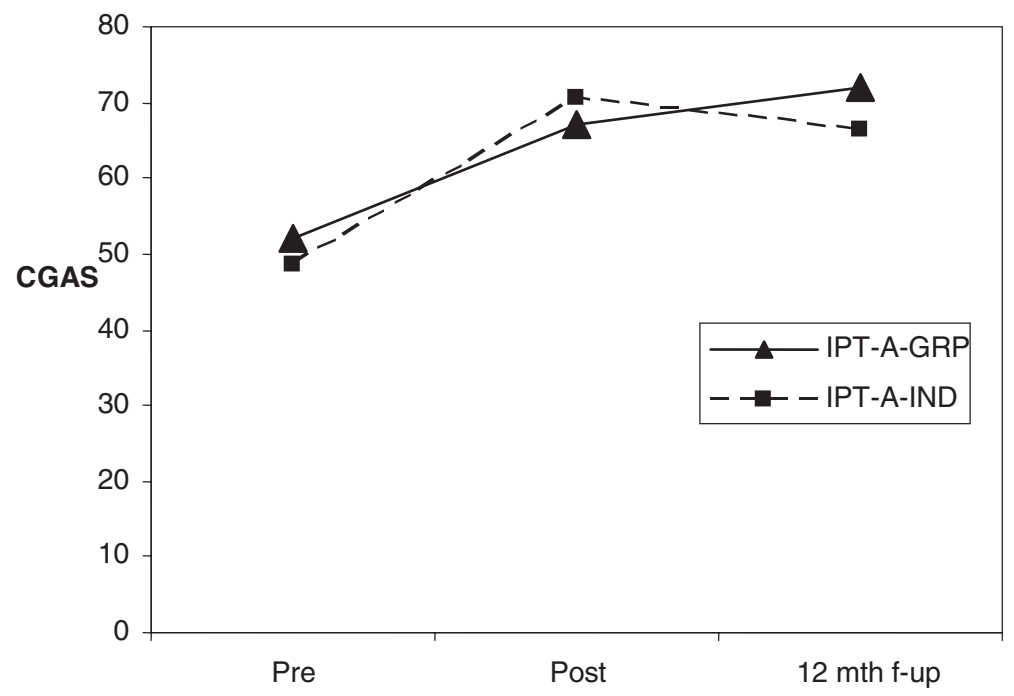

Figure 2. (a) BDI-II scores for ITT sample from pre-treatment to 12-month follow-up. (b) CGAS scores for ITT sample from pretreatment to 12-month follow-up 
Table 2. Values for diagnostic status and outcome variables for the completer sample across time and condition

\begin{tabular}{|c|c|c|c|c|c|c|c|}
\hline \multirow{2}{*}{$\begin{array}{l}\text { Treatment condition } \\
\text { Measures }\end{array}$} & \multirow[b]{2}{*}{ Time } & \multicolumn{3}{|c|}{ IPT-A-GRP } & \multicolumn{3}{|c|}{ IPT-A-IND } \\
\hline & & $n$ & $N$ & $\%$ & $n$ & $N$ & $\%$ \\
\hline \multirow[t]{5}{*}{ Free of MDD diagnosis } & Pre & 20 & 0 & 0 & 15 & 0 & 0 \\
\hline & Post $^{1}$ & 20 & 18 & 90.0 & 15 & 14 & 93.3 \\
\hline & Post $^{2}$ & 19 & 17 & 89.5 & 11 & 10 & 90.9 \\
\hline & 12-mth follow-up & 19 & 15 & 78.9 & 11 & 10 & 90.9 \\
\hline & & $n$ & $M$ & $S D$ & $n$ & $M$ & $S D$ \\
\hline \multirow[t]{4}{*}{ BDI-II } & Pre & 20 & 27.51 & 9.20 & 14 & 24.44 & 11.56 \\
\hline & Post $^{1}$ & 20 & 17.57 & 13.79 & 14 & 10.43 & 12.02 \\
\hline & Post $^{2}$ & 19 & 17.65 & 14.16 & 12 & 9.33 & 10.45 \\
\hline & 12-mth follow-up & 19 & 12.99 & 14.13 & 12 & 8.42 & 8.20 \\
\hline \multirow[t]{4}{*}{ CGAS } & Pre & 20 & 51.90 & 10.95 & 15 & 50.60 & 12.53 \\
\hline & Post $^{1}$ & 20 & 68.45 & 12.54 & 15 & 80.40 & 9.49 \\
\hline & Post $^{2}$ & 19 & 67.84 & 12.58 & 11 & 80.36 & 10.94 \\
\hline & 12-mth follow-up & 19 & 73.42 & 18.93 & 11 & 79.91 & 8.84 \\
\hline \multirow[t]{4}{*}{ CBCL-Int } & Pre & 19 & 19.32 & 8.05 & 15 & 19.39 & 9.54 \\
\hline & Post $^{1}$ & 19 & 18.62 & 7.76 & 15 & 13.13 & 8.59 \\
\hline & Post $^{2}$ & 18 & 18.15 & 7.71 & 12 & 11.92 & 6.71 \\
\hline & 12-mth follow-up & 18 & 16.70 & 9.19 & 12 & 7.17 & 6.49 \\
\hline \multirow[t]{4}{*}{ YSR-Int } & Pre & 20 & 27.10 & 11.01 & 14 & 27.51 & 10.49 \\
\hline & Post $^{1}$ & 20 & 20.85 & 11.27 & 14 & 16.57 & 13.64 \\
\hline & Post $^{2}$ & 19 & 20.74 & 11.57 & 12 & 15.92 & 12.14 \\
\hline & 12-mth follow-up & 19 & 18.47 & 14.25 & 12 & 16.64 & 9.97 \\
\hline \multirow[t]{4}{*}{ SCAS } & Pre & 20 & 41.36 & 13.74 & 14 & 50.59 & 14.03 \\
\hline & Post $^{1}$ & 20 & 35.61 & 14.34 & 14 & 37.09 & 15.27 \\
\hline & Post $^{2}$ & 19 & 35.33 & 14.67 & 12 & 35.35 & 12.98 \\
\hline & 12-mth follow-up & 19 & 34.66 & 20.39 & 12 & 34.92 & 11.30 \\
\hline \multirow[t]{4}{*}{ Comorbid diagnoses } & Pre & 20 & 1.05 & 1.14 & 15 & 0.80 & 0.94 \\
\hline & Post $^{1}$ & 20 & 0.50 & 0.95 & 15 & 0.33 & 0.72 \\
\hline & Post $^{2}$ & 19 & 0.53 & 0.96 & 11 & 0.09 & 0.30 \\
\hline & 12-mth follow-up & 19 & 0.47 & 1.07 & 11 & 0.00 & 0.00 \\
\hline
\end{tabular}

Notes: Post $^{2}=$ post-treatment data for those present at pre- and post-treatment; Post ${ }^{2}=$ post-treatment data for those present at post-treatment and 12-month follow-up; BDI-II $=$ Beck Depression Inventory - II; CBCL-Int $=$ Child Behaviour Checklist - Internalizing subscale; YSR-Int $=$ Youth Self Report - Internalizing subscale; SCAS = Spence Children's Anxiety Scale; CGAS = Children's Global Assessment of Functioning. IPT-A-GRP $=$ Group delivery of IPT for adolescents; IPT-A-IND = Individual delivery of IPT for adolescents.

Pre- to posttreatment. The pre-to post-treatment completer analyses included those individuals who were present at both of these measurement occasions. At post-treatment, 14 of $15(93.3 \%)$ completers in the IPT-A-IND condition and 18 of $20(90.0 \%)$ in the IPT-A-GRP condition no longer met criteria for MDD diagnosis, with no significant difference between conditions, $\chi 2(1, \mathrm{~N}=35)=0.12, p=.727$. Univariate analyses from pre to posttreatment revealed a significant time effect for the BDI-II, $\mathrm{F}(1,32)=27.95, \mathrm{p}<.001, \eta^{2}=.466$, CGAS, 
$\mathrm{F}(1,33)=144.22, p<.001, \eta^{2}=.814$, YSR-Int, $\mathrm{F}(1,32)=27.53, p<.001, \eta^{2}=.463$, CBCL-Int, $\mathrm{F}(1,32)=8.49, p=.006, \eta^{2}=.210$, SCAS-C, $\mathrm{F}(1,32)=20.92, p<.000, \eta^{2}=$ .395 , and number of comorbid diagnoses, $\mathrm{F}(1,33)=8.94, p=.005, \eta 2=.213$. There were no significant condition effects for any of these measures, but there were significant condition by time effects for the CBCL-Int, $\mathrm{F}(1,32)=5.43, p=.026, \eta^{2}=.145$, and CGAS, $\mathrm{F}(1,33)=$ $11.79, p=.002, \eta^{2}=.263$. Figures $3 \mathrm{a}$ and $3 \mathrm{~b}$ show the mean change over time for the BDI-II and CGAS respectively for the completer sample.

Post hoc contrasts for the CGAS showed significant improvements for both IPT-A-GRP and IPT-A-IND from pre- to post-treatment, $\left(\mathrm{F}(1,19)=39.03, p<.001, \eta^{2}=.673 ; \mathrm{F}(1,14)=\right.$ $120.59, p<.001, \eta^{2}=.896$ respectively). Although both conditions improved significantly on the CGAS, posttreatment scores were significantly higher (better) in the IPT-A-IND condition than the IPT-GRP condition, $\mathrm{F}(1,33)=9.51, p=.004, \eta^{2}=.224$ whereas there were no significant differences in CGAS between conditions at pretreatment (see Figure $3 b$ ).

Post hoc contrasts from pre- to post-treatment for the CBCL-Int indicated that, whereas IPT-A-IND showed a significant reduction in scores over time, $\mathrm{F}(1,14)=9.65, p<.001$, $\eta^{2}=.408$, there was no significant change for IPT-A-GRP, $\mathrm{F}(1,18)=.24, p=.627, \eta^{2}=$ .013 .

Posttreatment to 12-month follow-up. The posttreatment to 12-month follow-up completer analyses was limited to those individuals who were present at both of these measurement occasions. At 12-month follow-up, 10 of $11(90.9 \%)$ of those in the IPT-AIND condition and 15 of $19(78.9 \%)$ of those in IPT-A-GRP condition no longer held a MDD diagnosis, with no significant difference between conditions, $\chi^{2}(1, \mathrm{~N}=30)=0.718, p=$ .397. From posttreatment to 12-month follow-up, there were no significant time, condition, or condition by time effects for the BDI-II, YSR-Int, SCAS-C or number of comorbid diagnoses, suggesting maintenance of improvements over time for both conditions on these measures.

For the CGAS, there were no significant time nor condition by time effects, but a significant effect for condition was evident, $\mathrm{F}(1,28)=4.33, p<.05, \eta^{2}=.134$. This suggested a general tendency for the IPT-A-IND condition to show higher (better) CGAS scores than the IPT-AGRP condition during the posttreatment to 12-month follow-up period. However, as shown in Figure 3b, the IPT-A-GRP condition continued to improve during the 12-month followup whereas the IPT-A-IND condition remained static and there was no longer a significant difference in CGAS scores between conditions at 12-month follow-up.

For the CBCL-Int, there were significant effects for time, $F(1,28)=5.67, p<.05, \eta^{2}=$ .168 , and condition, $F(1,28)=9.22, p=.005, \eta^{2}=.248$, but not for condition by time. The significant time effect suggests that participants in general tended to show reductions on the CBCL-Int during the 12-month follow-up (see Table 2). The significant condition effect reflects the tendency for those in the IPT-A-IND condition to report lower CBCLInt scores than those in the IPT-A-GRP condition during the follow-up period in general, reflecting maintenance of the reduction in parent-reported internalizing evident from pre- to post-treatment in the IPT-A-IND condition.

Taken together, these results suggest that, for the completer sample, those receiving IPT showed improvements in depression, anxiety, youth-reported internalizing problems, clinician-rated global functioning, and number of comorbid diagnoses from pre- to posttreatment. These improvements were maintained at 12-month follow-up. There were no significant differences between group and individual therapy formats in the extent of changes 
(a)

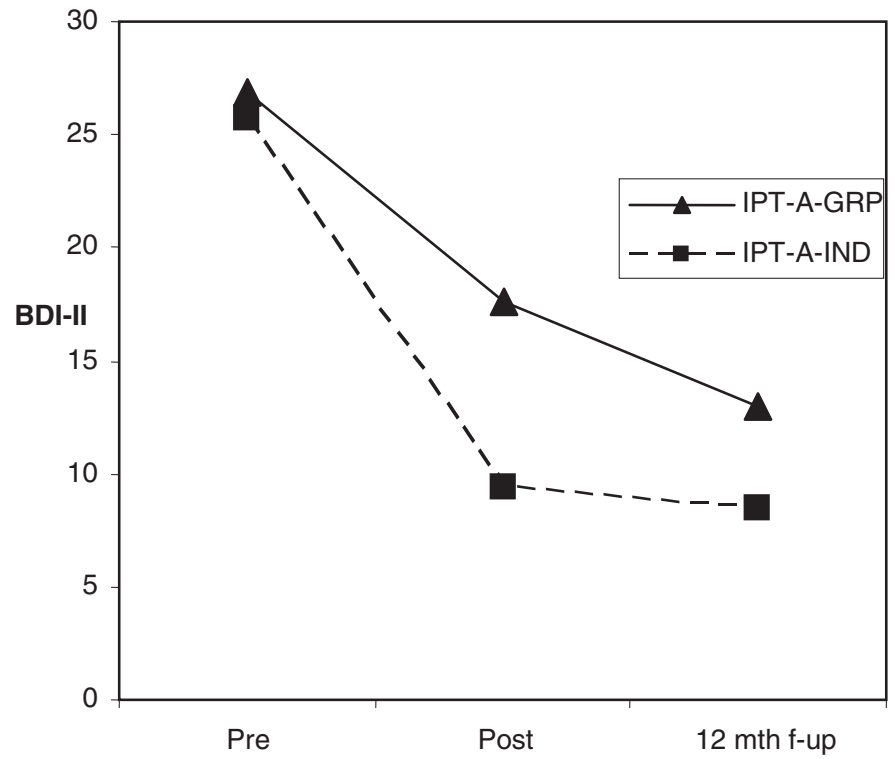

(b)

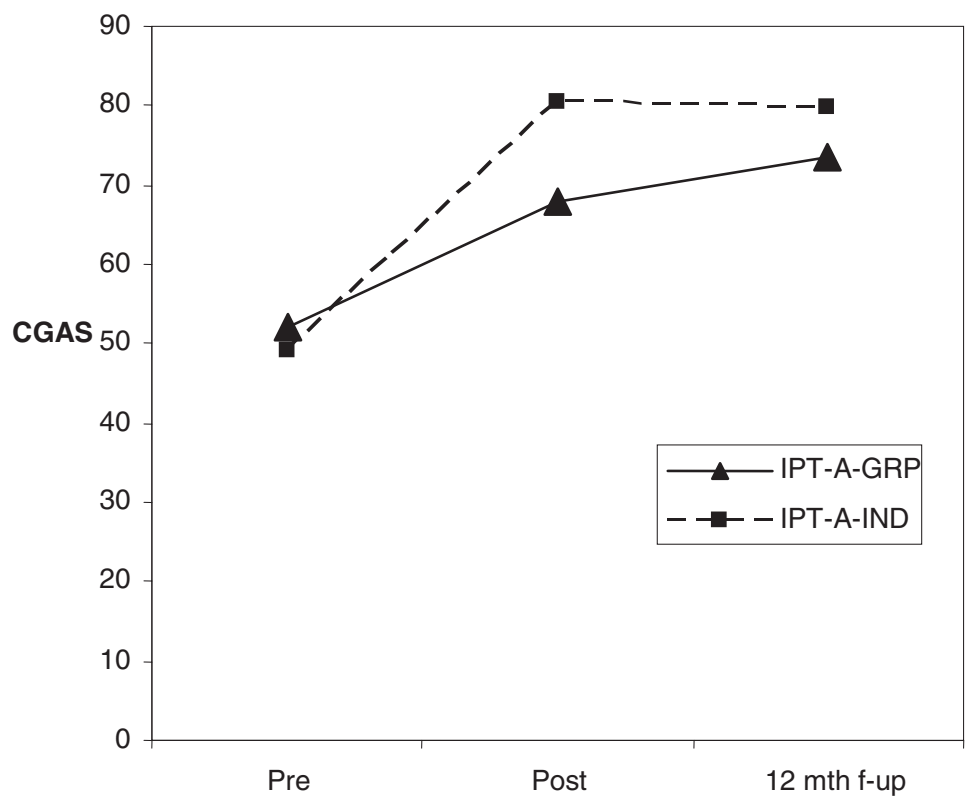

Figure 3. (a) BDI-II scores for completer sample from pre-treatment to 12-month follow-up. (b) CGAS scores for completer sample from pretreatment to 12-month follow-up 
over this period on any of the measures except for parent-reported internalizing problems and clinician-rated global functioning. Individual IPT was associated with significantly greater improvements than group IPT in terms of parent-reported internalizing problems and clinician-rated global functioning from pre- to posttreatment for the completer sample. From posttreatment to 12-month follow-up, those who received individual IPT in the completer sample continued to show lower levels of parent-reported internalizing problems and higher clinician ratings of global functioning than those who received group IPT, although the extent of this difference decreased on the clinician ratings of global functioning over this period.

\section{Discussion}

In terms of the first hypothesis that adolescents receiving both IPT-A-GRP and IPT-A-IND would show significant improvement from pre- to posttreatment, both ITT and completer analyses indicated that the majority of adolescents no longer met full criteria for a MDD diagnosis at posttreatment, with no significant difference between delivery formats on this outcome measure. In addition, participants in both the ITT and completer conditions demonstrated significant reductions in depression severity, youth-reported internalizing problems, anxiety, and number of comorbid diagnoses and improvements in global functioning. The ITT analyses did not indicate significant differences in outcome between treatment formats on any of the outcome measures. For the completer sample, however, those in IPT-A-IND showed significantly greater improvements than those in IPT-A-GRP from pre- to posttreatment on the measure of therapist-rated global functioning. The IPT-A-IND participants also showed significant reductions in parent-reported internalizing problems from pre- to posttreatment, whereas IPT-A-GRP participants showed minimal change.

There was only slight support for the second hypothesis that IPT-A-IND would be associated with more sustained treatment benefits over the 12-month follow-up period than IPT-A-GRP. For diagnostic status, depressive symptoms, anxiety, youth-reported internalizing problems, therapist-rated global functioning, and number of comorbid diagnoses there were no significant differences between treatment conditions in terms of changes from posttreatment to 12-month follow-up for either the ITT or completer analyses. Both treatments tended to maintain improvements over this period. However, for parent-reported internalizing problems, the significant reduction evident in the IPT-A-IND condition at posttreatment was maintained at 12-month follow-up in the completer sample, and those in the IPT-A-IND condition continued to report lower scores on this measure than those in the IPT-A-GRP condition over the follow-up period.

Given the higher attrition rate for the IPT-A-IND condition, it is difficult to interpret the difference between group and individual treatment delivery for the completer sample on parent report of their child's internalizing problems, an effect that was not evident in the ITT analyses. On the one hand, it is possible that adolescents in the IPT-A-IND condition who did not participate in posttreatment and 12-month assessments may have been those who did not respond well to treatment, and thus the results for the completer sample may have overestimated the improvements for the IPT-A-IND condition. On the other hand, it is possible that use of the LOCF method for the ITT analyses (replacing missing values with those from the previous assessment) may have underestimated the extent of reductions in the IPT-A-IND therapy condition for the ITT analyses. There was no reason for the differential dropout across 
treatments that could be identified. Nevertheless, the differential attrition makes it difficult to draw firm conclusions about differences in outcome across treatment formats.

In general, the results demonstrated strong and significant improvements in outcome for both IPT-A-IND and IPT-A-GRP for adolescents. In particular, it should be noted that both depression outcome measures (clinical MDD diagnosis and youth-reported depression) showed equivalent improvements for both formats of therapy for both the ITT and completer samples, and that these effects were maintained at 12-month follow-up.

\section{Clinical significance and implications of the results}

The finding that, in the completer sample, $78.9 \%$ of adolescents who received IPT-A-GRP and $90.9 \%$ who received IPT-A-IND no longer met full criteria for MDD at 12-month follow-up is comparable to the results found in a recent evaluation of IPT with adults (Ravitz, Maunder and McBride, 2008) where $84.9 \%$ of treatment completers experienced full or partial remission of their MDD diagnosis. In terms of the severity of the presenting problem at baseline, the sample in the present study is similar to that reported elsewhere in the literature. The mean baseline CGAS rating was 50.15 across the sample, which is similar to that of 52.6 reported by Mufson, Dorta et al. (2004) and 52.0 reported by Mufson et al. (1999) with adolescents, and indicative of a moderate level of impairment (Shaffer et al., 1983). By posttreatment, the mean ITT CGAS rating was 66.95 in the present study for IPT-A-GRP and 70.47 for IPTA-IND, again consistent with the CGAS outcome of 66.7 reported by Mufson, Dorta et al. (2004) in their ITT sample, and indicative of only slight impairment (Shaffer et al.,1983).

Although the findings should be treated with caution given the limited sample size and other methodological limitations summarized below, the results provide tentative support for the use of IPT-A-GRP with adolescents. The group approach was associated with a high retention rate and clinically significant outcomes. Given the cost savings of groupbased treatment, the approach may offer promise in the delivery of mental health services for depressed adolescents. Future studies should examine client satisfaction and participant perceptions of group versus individual delivery of IPT-A to determine whether young people have a preference for, or perceive greater benefits from, individual versus group delivery.

Certainly, the study adds to the limited body of evidence concerning the benefits of IPT in the treatment of adolescent depression, and it appears to provide a valid alternative to CBT. Future studies should investigate whether there are individual characteristics of depressed young people that predict superior outcome from IPT versus CBT so as to match young people to the most appropriate treatment approach.

\section{Strengths and limitations}

The present study had several strengths. The sample consisted of clinically depressed adolescents who had a DSM-IV diagnosis of MDD ascertained by standardized clinical interview, with outcome being assessed through re-administration of the structured interview. Previous studies have typically relied on self-report measures of depression rather than a clinician-administered clinical interview (e.g. Rosselló and Bernal, 1999). The current study also avoided the stringent inclusion and exclusion criteria that characterize many efficacy studies. Most of the participants had comorbid disorders such as dysthymia, bulimia nervosa, 
alcohol dependence, substance abuse, oppositional defiant disorder, and anxiety disorders. As such, the results are generalizable to many clinic situations in which young people present. Another strength of the study was the significant attention paid to the training and supervision of IPT therapists. Although adherence to treatment manuals was not measured, the high level of training and supervision provided is likely to have led to increased therapist skill and treatment adherence.

Nevertheless, there were methodological issues that limit the conclusions that can be drawn. As noted above, the differential dropout rates across conditions makes it difficult to draw firm conclusions about differences in outcome across treatment formats. In addition, the relatively small sample size provides limited power to detect significant effects between treatment formats. Ideally, a much larger sample should be used in comparing outcomes across treatments if the aim is to detect relatively small differences in effect sizes across conditions. The present study also failed to include a wait-list control group. Although initially intended as part of the study design, parents and medical practitioners were extremely reluctant to allow adolescents to remain on a wait-list. The use of a treatment-as-usual comparison would offer a useful alternative approach in future studies.

An additional limitation was that the design of the present study did not enable a comparison of dosages across experimental conditions. On the one hand, the group sessions were longer in duration than the individual sessions. On the other hand, the inclusion of multiple participants in a group creates less time for each individual to focus on their own issues in the group compared to individual treatment formats. It would be valuable in future studies to try to disentangle these elements of therapy and to determine their association with treatment outcome.

The lack of quantitative assessment of therapist compliance with the treatment protocols is also a limitation, although strong efforts were made to ensure high quality training and supervision of therapists in order to optimize treatment fidelity. The lack of inter-assessor reliability checks for diagnoses in the clinical interviews also weakened the study design. Finally, the study would have been strengthened by more rigorous counterbalancing of therapists across conditions. Although this is unlikely to have impacted upon the results given that two of the therapists conducted the three groups and the majority of individual cases, it would have been preferable if counterbalancing had been perfect.

\section{Conclusion}

In conclusion, the results of this small clinical trial add to the very limited body of knowledge concerning the long-term efficacy of IPT in the treatment of depression in adolescents. Both individual and group forms of IPT were associated with significant and sustained reductions in depression and associated symptoms and improvements in global functioning. There was only slight support that individual delivery produced significantly greater long-term effects than group delivery, with this effect being limited to the completer sample and to parent report of internalizing problems. Indeed, group delivery of IPT was associated with significant improvements on the majority of outcome measures in both the completer and ITT samples. Thus, IPT-A-GRP offers promise as a cost-effective approach to the delivery of treatment for depression among adolescents and warrants further investigation in larger research trials. 


\section{References}

Achenbach, T. M. (1991). Integrative Guide to the 1991 CBCL/4-18, YSR, and TRF Profiles. Burlington, VT: University of Vermont, Department of Psychology.

Achenbach, T. M., Dumenci, L. and Rescorla, L. A. (2002). Ten-year comparisons of problems and competencies for national samples of youth: self, parent and teacher reports. Journal of Emotional and Behavioral Disorders, 10, 194-203.

Ambrosini, P. J., Metz, C., Bianchi, M. D. and Rabinovich, H. (1991). Concurrent validity and psychometric properties of the Beck Depression Inventory in outpatient adolescents. Journal of the American Academy of Child and Adolescent Psychiatry, 30, 51-57.

American Psychiatric Association (1994). Diagnostic and Statistical Manual of Mental Disorders (4th ed.). Washington, DC: American Psychiatric Publishing, Inc.

Atlas, J. A. and DiScipio, W. J. (1992). Correlations of Beck Depression Inventory and Reynolds Adolescent Depression Scale. Psychological Reports, 70, 621-622.

Barrera, M. and Garrison-Jones, C. V. (1988). Properties of the Beck Depression Inventory as a screening instrument for adolescent depression. Journal of Abnormal Child Psychology, 16, 263273.

Beck, A. T., Steer, R. A. and Brown, G. K. (1996). Manual for Beck Depression Inventory - II. San Antonio, TX: The Psychological Corporation.

Hill, C. E., O'Grady, K. E. and Elkin, I. (1992). Applying the Collaborative Study Psychotherapy Rating Scale to Rate Therapist Adherence in Cognitive Behavior Therapy, Interpersonal Therapy, and Clinical Management. Journal of Consulting and Clinical Psychology, 60, 73-79.

Kaufman, J., Birmaher, B., Brent, D. and Rao, U. (1997). Schedule for Affective Disorders and Schizophrenia for School-Age Children-Present and Lifetime version (K-SADS-PL): initial reliability and validity data. Journal of the American Academy of Child and Adolescent Psychiatry, 36, 980-988.

Kieling, C., Baker-Henningham, H., Belfer, M., Conti, G., Ertem, I., Omigbodun, O., et al. (2011). Child and adolescent mental health worldwide: evidence for action. The Lancet, 378(9801), 15151525.

Klein, D. N., Dougherty, L. R. and Olino, T. M. (2005). Toward guidelines for evidence-based assessment of depression in children and adolescents. Journal of Clinical Child and Adolescent Psychology, 34, 412-432.

Klerman, G. L., Dimascio, A., Weissman, M., Prusoff, B. and Paykel, E. S. (1974). Treatment of depression by drugs and psychotherapy. American Journal of Psychiatry, 131, 186-191.

Kumar, G., Steer, R. A., Teitelman, K. B. and Villacis, L. (2002). Effectiveness of Beck Depression Inventory-II subscales in screening for major depressive disorders in adolescent psychiatric inpatients. Assessment, 9, 164-170.

Mufson, L., Dorta, K. P., Wickramaratne, P., Nomura, Y., Olfson, M. and Myrna, M. W. (2004). A randomized effectiveness trial of Interpersonal Psychotherapy for Depressed Adolescents. Archives of General Psychiatry, 61, 577-584.

Mufson, L., Gallagher, T., Dorta, K. P. and Young, J. F. (2004). A group adaptation of Interpersonal Psychotherapy for Depressed Adolescents. American Journal of Psychotherapy, 58, 220-237.

Mufson, L., Moreau, D., Weissman, M. M. and Klerman, G. L. (1993). Interpersonal Psychotherapy for Depressed Adolescents. New York: Guilford Press.

Mufson, L., Weissman, M. M., Moreau, D. and Garfinkel, R. (1999). Efficacy of interpersonal psychotherapy for depressed adolescents. Archives of General Psychiatry, 56, 573-579.

Muris, P., Schmidt, H. and Merckelbach, H. (2000). Correlations among two self-report questionnaires for measuring DSM-defined anxiety disorder symptoms in children: the Screen for Child Anxiety Related Emotional Disorders and the Spence Children's Anxiety Scale. Personality and Individual Differences, 28, 333-346. 
Puig-Antich, J. and Chambers, W. (1978). Schedule for Affective Disorders and Schizophrenia for School Aged Children, Epidemiological Version. K-SADS-E (3rd version).

Ravitz, P., Maunder, R. and McBride, C. (2008). Attachment, contemporary interpersonal theory and IPT: an integration of theoretical, clinical, and empirical perspectives. Journal of Contemporary Psychotherapy, 38, 11-21.

Rosselló, J. and Bernal, G. (1999). The efficacy of cognitive-behavioral and interpersonal treatments for depression in Puerto Rican adolescents. Journal of Consulting and Clinical Psychology, 67, 734745.

Rosselló, J., Bernal, G. and Rivera-Medina, C. (2008). Individual and group CBT and IPT for Puerto Rican adolescents with depressive symptoms. Cultural Diversity and Ethnic Minority Psychology, 14, 234-245.

Rounsaville, B. J., Chevron, E. S., Weissman, M., Prussof, B. A. and Frank, E. (1986). Training therapists to perform interpersonal psychotherapy in clinical trials. Comprehensive Psychiatry, 27, 364-371.

Shaffer, D., Gould, M. S., Brasic, J., Ambrosini, P., Fischer, P., Bird, H., et al. (1983). A Children's Global Assessment Scale. Archives of General Psychiatry, 40, 1228-1231.

Spence, S. H. (1998). A measure of anxiety symptoms among children. Behaviour Research and Therapy, 36, 545-566.

Spence, S. H., Barrett, P. M. and Turner, C. M. (2003). Psychometric properties of the Spence Children's Anxiety Scale with young adolescents. Journal of Anxiety Disorders, 17, 605-625.

Strober, M., Green, J. and Carlson, G. (1981). Utility of the Beck Depression Inventory with psychiatrically hospitalized adolescents. Journal of Consulting and Clinical Psychology, 49, 482483.

Wilfley, D. E., MacKenzie, K. R., Welch, R. R., Ayres, A. E. and Weissman, M. (2000). Interpersonal Psychotherapy for Group. New York: Basic Books. 